\title{
Predictive Value of Preoperative MRI Using the \#ENZIAN Classification Score in Patients with Deep Infiltrating Endometriosis
}

\author{
Aysun FENDAL TUNCA ( $\square$ aysunfendaltunca@gmail.com ) \\ University of Health Sciences Istanbul https://orcid.org/0000-0001-8906-8586 \\ Derya Ece Iliman \\ University of Health Sciences Istanbul https://orcid.org/0000-0002-0409-4089 \\ Aysegul Akdogan Gemici \\ University of Health Sciences Istanbul https://orcid.org/0000-0002-7707-1849 \\ Cihan Kaya \\ Acibadem Mehmet Ali Aydinlar University https://orcid.org/0000-0003-4175-7694
}

\section{Research Article}

Keywords: \#ENZIAN Classification, Deep Infiltrating Endometriosis, MRI, Endometriosis Imaging

Posted Date: December 8th, 2021

DOI: https://doi.org/10.21203/rs.3.rs-1001165/v1

License: (c) (i) This work is licensed under a Creative Commons Attribution 4.0 International License. Read Full License 


\section{Abstract \\ Purpose}

The aim of this study is to investigate the correlation between the magnetic resonance imaging (MRI) and intraoperative findings of deep infiltrating endometriosis using the \#ENZIAN score.

\section{Methods}

This retrospective study included 64 patients who underwent surgery for deep infiltrating endometriosis between January 2017 and August 2020. Preoperative abdominopelvic MRI assessment was evaluated and scored using the \#ENZIAN classification. Operative scores were considered the gold standard, and the sensitivity, specificity, and positive and negative predictive values (PPV and NPV) of MRI for each category were calculated.

\section{Results}

MRI has higher sensitivity and specificity in showing the lesions of the compartments 0 (ovarian lesions), A (rectovaginal septum and posterior vaginal fornix), and B (uterosacral ligaments and parametrium) $(100-100 \%, 100-100 \%$, and $97-100 \%$, respectively, $p<0.001$ ) compared to the other compartments. The lowest sensitivity, specificity, accuracy, and PPV of the MRI was found in compartment P $(14 \%, 76 \%, 70 \%$, and $7 \%$, respectively).

\section{Conclusion}

We demonstrated that the \#ENZIAN classification in MRI reports has significant sensitivity and specificity in compartments A, B (uterosacral ligaments and parametrium), and $\mathrm{O}$. Furthermore, the determination of peritoneal lesions via MRI is inadequate.

\section{Introduction}

Endometriosis is a benign chronic inflammatory disease characterized by the presence of endometrial tissue outside the uterus. Endometriosis affects $5-10 \%$ of women of reproductive age [1] It typically occurs in ovaries, tubes, uterosacral ligaments, bowels, ureters, bladder, and rarely lungs, brain, and scars. Despite the different locations of the disease, dysmenorrhea, dyspareunia, dyschezia, pelvic pain, and infertility are common symptoms [2]. Even though the incidence of endometriosis is high, late diagnosis is common. It takes about 6-11 years from first symptoms to diagnosis due to the limitations of clinical experience and underestimation of the symptoms, especially in the adolescent population, and the lack of expertise in endometriosis sonography[3-4-5].

Thus, magnetic resonance imaging (MRI) is a powerful non-invasive tool that provides an evaluation of the upper GI lesions for preoperative surgical mapping. In 2014, Mederios et al. published a meta-analysis in which the overall sensitivity and specificity of pelvic MRI was $83 \%$ and $90 \%$, respectively [6]. However, laparoscopy remains the gold standard for the diagnosis of the disease [1-6].

Endometriosis is a condition that requires the involvement of varied specialists in both preoperative and intraoperative care. Even though transvaginal ultrasound (TVS) is more affordable and more easily accessible than MRI as a diagnostic tool, performing TVS on endometriosis patients requires a significant amount of expertise. MRI is widely used for this reason; therefore, radiologists must have some knowledge of the surgical process. Similarly, surgeons are also a substantial part of the diagnosis in terms of examination, TVS, and laparoscopy and should also be familiar with MRI. To be able to facilitate these relations, a standardized MRI reporting system is crucial. Classification of endometriosis is complicated, and in 2014, the World Endometriosis Society proposed a classification toolbox that includes the rASRM classification, the ENZIAN classification, and the Endometriosis Fertility Index (EFI) [7]. However, using multiple classifications in daily practice is not practical and may not be used effectively. In 2021, peritoneal, tubal, and ovarian involvement and pelvic adhesions were added to the current ENZIAN score, and it was renamed as the \#ENZIAN classification [8]. Since it is a new classification, no study has been published comparing the relation between MRI and \#ENZIAN score considering intraoperative findings. The \#ENZIAN classification has undergone multiple revisions since its first appearance to become a more suitable system, and as it gets more widely used, it may be used as a standard reporting tool for MRI.

This study aimed to compare the preoperative MRI and \#ENZIAN scores in the prediction of intraoperative findings.

\section{Material And Methods}


This retrospective study consists of a total of 64 cases, which were all diagnosed with deep infiltrating endometriosis (DIE) and operated in a tertiary referral center between January 2017 and August 2020. The inclusion criteria were the presence of preoperative abdominopelvic MRI assessment and postoperative histologic DIE verification. The exclusion criteria were missing sociodemographic data and MRI and/or malignancy.

All surgeries were performed by the same surgical team, and all operations were completed laparoscopically. Video documentation is a must in this center; therefore, videos were available for all cases. The primary surgeon evaluated all case videos, and the \#ENZIAN score was determined considering intraoperative findings (Fig. 1).

The \#ENZIAN score is a revised classification of the ENZIAN score. In addition to the ENZIAN score, peritoneal, ovarian, and tubo-ovarian compartments are assessed in the \#ENZIAN score system, which consists of six main compartments. These compartments are as follows: $P$ (peritoneal lesions $<5 \mathrm{~mm}$ ), O (all endometriomas and infiltration ovarian surface foci $>5 \mathrm{~mm}$ ), $\mathrm{T}$ (tubo-ovarian condition $+/$ - tubal patency), A (craniocaudal axis, rectovaginal septum, and posterior vaginal fornix), B (mediolateral axis, uterosacral ligaments (USL), and parametrium), and $\mathrm{C}$ (ventrodorsal axis and anterior wall of the rectum). Compartments $\mathrm{P}$ and $\mathrm{O}$ are subdivided by the sum of all lesions and compose a virtual diameter: 1 is $<3 \mathrm{~cm}, 2$ is $3-7 \mathrm{~cm}$, and 3 is $>7 \mathrm{~cm}$. T is subdivided into three categories according to the site of adhesions of the adnexa: 1 is to pelvic sidewall or tubo-ovarian, 2 is adhesions to the uterus, and 3 is adhesions to USL or bowel. A, B, C compartments are subdivided into three categories as well by the extent of lesions as follows: $1(<1 \mathrm{~cm}), 2(1-3 \mathrm{~cm})$, and $3(>3 \mathrm{~cm})$. Uterine and extragenital locations are also described as FA (adenomyosis), FB (bladder involvement), FU (ureter involvement with signs of obstruction), and FI (bowel involvement cranial to the rectosigmoid junction) [8].

\section{MRI Technique}

All MRI examinations were performed on a 1.5T Avanto fit Siemens MR unit (Siemens Healthcare, Erlangen, Germany) using phase-array eightchannel pelvic coil (Siemens Healthcare, Erlangen, Germany). Antiperistaltic agents and vaginal or rectal contrast medium were not used. Also, no bowel preparation was done for any patient.

Each measurement included axial T1-weighted sequences with and without fat suppression. T2-weighted sequences were obtained from axial, sagittal, and coronal planes without a fat saturation. The standard gynecology setting included the whole pelvis from iliac crests to the pubic bone. The slice thickness was 3-4 mm with interslice gaps of $0.0 \mathrm{~mm}-1.0 \mathrm{~mm}$.

In addition, dynamic contrast-enhanced sequences containing axial T1-weighted three-dimensional (3D) fast spoiled gradient recall echo sequences were performed. Gadolinium-diethylene triamine pentaacetic acid (Magnevist; Bayer HealthCare, Wayne, NJ) as contrast material was injected at a dose of $0.1 \mathrm{mmol} / \mathrm{kg}$ body weight using an automated pump (Nemoto; Nemoto Kyorindo, Tokyo, Japan) followed by a 20-mL saline flush, both at a rate of $2 \mathrm{~mL} / \mathrm{s}$. The sequences matched the recommendations of the European Society of Urogenital Radiology (ESUR) guidelines [9].

The records of patients who underwent DIE operation in the tertiary center between 2017 and 2020 were independently evaluated and scored by a senior radiologist. Each compartment of the \#ENZIAN classification were assessed and compared by both parties. Operative scores were considered the gold standard, and sensitivity, specificity, and positive and negative predictive values (PPV and NPV, respectively) of the MRI for each category were calculated (Fig. 2)

\section{Statistical Analysis}

The data was described as numbers and percentages. Statistical evaluation was performed using the SPSS (version 20.0; SPSS Inc., Chicago, IL, USA) software. Fisher's exact or Chi-square test was performed for categorical data, and the Kruskal-Wallis test was performed for continuous variables. Surgical results were accepted as standard and preoperative MRI findings were compared. Sensitivity, specificity, PPV, NPV, accuracy, precision, and association (k-Cohen coefficient) scores were calculated. The scores for the compartments were also calculated using Kendall's tau b test according to their subdivision (size 1-3). Data for compartments A, B, and C were separated into A0 (respectively, B0, $\mathrm{C} 0$, for no lesion) and A1 (respectively, B1, C1 for any lesion) and compared to each other. $P$ values less than 0.05 were accepted as statistically significant (two-tailed).

\section{Results}

Sensitivity, specificity, PPV, and NPV of all main compartments and the FA compartment are presented in Table 1. FB, FU, and FI were excluded due to low number of cases. Three cases had intraoperative FI involvement, whereas one case had FB involvement. MRI classification showed one FB and eleven FI involvement, and of those, only one case was reported to have FI involvement intraoperatively. 
Table 1

Comparison of the preoperative MRI findings with the intraoperative findings considering the \#ENZIAN classification

\begin{tabular}{|c|c|c|c|c|c|c|c|c|c|c|}
\hline Surgery & MRI + & MRI - & Specificity(\%) & Sensitivity(\%) & Accuracy(\%) & PPV(\%) & NPV(\%) & $\begin{array}{l}\text { Kendal } \\
\text { Tau b }\end{array}$ & $\begin{array}{l}\text { Cohen } \\
\text { Kappa }\end{array}$ & ${ }_{\text {value }}^{p}$ \\
\hline$P+$ & $1(14.3 \%)$ & $13(23.2 \%)$ & 76 & 14 & 70 & 7 & 87 & 0.54 & 0.59 & 0.51 \\
\hline$P-$ & $6(85.7 \%)$ & $43(76.8 \%)$ & & & & & & & & \\
\hline $0+$ & $62(100 \%)$ & $0(0)$ & 100 & 100 & 100 & 100 & 100 & 0.31 & $<0.001$ & 0.01 \\
\hline $0-$ & $0(0)$ & $1(100 \%)$ & & & & & & & & \\
\hline$T+$ & $37(92.5)$ & $3(13.6 \%) \%)$ & 86 & 92 & 90 & 92 & 86 & $<0.001$ & $<0.001$ & $<0.001$ \\
\hline $\mathrm{T}-$ & $3(7.5 \%)$ & $19(86.4 \%)$ & & & & & & & & \\
\hline$A+$ & $4(100 \%)$ & $0(0)$ & 100 & 100 & 100 & 100 & 100 & 0.02 & $<0.001$ & $<0.001$ \\
\hline A- & $0(0)$ & $59(100 \%)$ & & & & & & & & \\
\hline$B+$ & $42(97.7 \%)$ & $0(0)$ & 100 & 97 & 98 & 100 & 95 & $<0.001$ & $<0.001$ & $<0.001$ \\
\hline B - & $1(2.3 \%)$ & $20(100 \%)$ & & & & & & & & \\
\hline$C+$ & $18(94.7 \%)$ & $2(4.5 \%)$ & 95 & 94 & 95 & 90 & 97 & $<0.001$ & $<0.001$ & $<0.001$ \\
\hline C- & $1(5.3 \%)$ & $42(95.5 \%)$ & & & & & & & & \\
\hline FA + & $8(88.9 \%)$ & $7(13 \%)$ & 87 & 88 & 87 & 53 & 97 & 0.001 & $<0.001$ & $<0.001$ \\
\hline FA- & $1(11.1 \%)$ & $47(87 \%)$ & & & & & & & & \\
\hline
\end{tabular}

MRI regarding the departments $\mathrm{O}, \mathrm{A}$, and B were the most sensitive and specific (100-100\%, 100-100\%, and 97-100\%, respectively) with $p$ value being $<0.001$, for all comparisons. Lowest sensitivity, specificity, accuracy, and PPV were calculated in compartment $\mathrm{P}(14 \%, 76 \%, 70 \%$, and $7 \%$, respectively). These calculations were made only by the presence of the lesions in each compartment. The number and size of the lesions were not taken into account, since these are not as critical as the lesion site in preoperative preparation (Table 1).

Intraoperative involvement evaluation included both more recent endometriotic foci with glandular, purple-red appearance and older lesions with fibrosis and adherent to the surrounding tissues.

\section{Discussion}

In this study, MRI and operative scores showed significant agreement regarding compartments $\mathrm{A}, \mathrm{B}, \mathrm{O}$, and $\mathrm{C}$, which was compatible with previous studies using the previous ENZIAN classification [10-12]. Our results demonstrated $100 \%$ specificity and sensitivity of MRI in the evaluation of rectovaginal septum and posterior vaginal fornix considering (compartment A) the \#ENZIAN classification.

DIE procedures are one of the most complicated operations for gynecological surgeons. It may result in multiple complications and comorbidities such as bowel, bladder, and ureter injuries and bleeding from adhesion sites. The overall rate of severe complications for DIE operations is reported to be 7-9\% [13-16]. Preoperative knowledge of the involved pelvic organs facilitates minimizing these complications as well as disease recurrence, providing an effective preoperative strategy via a multidisciplinary approach. Currently, TVS and MRI are effectively used in DIE diagnosis by experienced gynecologists and radiologists. TVS has $93 \%$ sensitivity and $96 \%$ specificity, whereas MRI has $95 \%$ sensitivity and $91 \%$ specificity in the diagnosis of DIE. Nonetheless, laparoscopy remains the gold standard for endometriosis diagnosis [17].

American Society of Reproductive Medicine (rASRM) classification has been the most widely used scoring system for defining the severity of the disease [18]. However, it does not define adherent organ involvement and the location of DIE lesions [19]. The relation between the rASRM classification and symptom severity has been evaluated in various studies; however, the lack of reliable correlation [20-23] has led physicians to seek a much more relevant classification model. The ENZIAN classification emerged for this purpose to provide a decent definition of DIE lesions. A correlation between ENZIAN and DIE symptoms was demonstrated in terms of location and severity [24, 25]. Moreover, ENZIAN demonstrated a correlation between DIE symptoms, the extent of the disease, and surgery length [25-27]. However, regardless of the intraoperative classification, MRI and TVS remain the most valuable preoperative diagnostic tools.

A meta-analysis evaluating the data of 20 studies reported an overall sensitivity and specificity of MRI for pelvic DIE diagnosis as $83 \%$ and $90 \%$, respectively [6]. In another study, Dipaola et al. compared MRI and intra-operative ENZIAN scores in 115 patients, revealing a significant concordance between histopathologic results of excised tissues in vagina-rectovaginal space, uterosacral ligaments, rectum-sigmoid colon, 
and adenomyosis and MRI scores with accuracy reported as $96 \%, 98 \%, 96 \%$, and $100 \%$, respectively [12]. In addition, Burla et al. evaluated the correlation between preoperative MRI and ENZIAN score. They reported $95.2 \%$ sensitivity and $95.7 \%$ specificity for compartment A [10], which was $100 \%$ in our results.

In terms of modifications in the \#ENZIAN classification, compartment B includes uterosacral ligaments (USL) and pelvic sidewall involvement. Besides, the \#ENZIAN classification evaluates right and left pelvic sides separately, which is in contrast to the previous revised ENZIAN classification [8]. Another difference between the revised ENZIAN classification and the \#ENZIAN classification is that in the latter, compartment $B$ does not include ureter involvement or hydronephrosis, which has an incidence ranging from $0.3-12 \%$ and is often asymptomatic[28]. The clinical significance of ureteral endometriosis has led to the need for a separate classification of the condition. In our study, MRI had $97 \%$ sensitivity and $100 \%$ specificity in compartment B. A meta-analysis evaluating the data of 20 studies showed $85 \%$ sensitivity and $80 \%$ specificity of MRI in terms of USL [6]. Dipaola et al. found $97 \%$ sensitivity and $99 \%$ specificity in compartment B [12]. In contrast, a study comparing the previous ENZIAN classification to MRI findings reported a $78 \%$ sensitivity and $100 \%$ specificity for compartment B [10]. The reason behind this low sensitivity is not apparent; however, we may speculate that the accuracy of MRI could decrease in severe cases and is also related to the experience.

Regarding compartment $\mathrm{C}$ in our study, high sensitivity and specificity were achieved at $95 \%$ and $94 \%$, respectively. It is worth mentioning that these results only include the rectum, which is described as a $16 \mathrm{~cm}$ segment of the colon adjacent proximally to the anal verge. In contrast to the rectosigmoid region, compartment $\mathrm{C}$ does not involve the sigmoid colon. A meta-analysis evaluating both rectum and sigmoid colon lesions reported $83 \%$ sensitivity and $88 \%$ specificity[7]. Two other studies evaluated MRI accuracy of DIE lesions using the revised ENZIAN score, and both reported $86 \%$ sensitivity in compartment C. Specificities of the compartment C in these studies were reported as $98 \%$ [12] and $89 \%[10]$.

Another improvement in \#ENZIAN is the inclusion of the tubo-ovarian condition compartment. In this compartment, tubal and ovarian adhesions to pelvic sidewall, uterus, USL, and bowels are assessed. TVS has $93 \%$ sensitivity and $96 \%$ specificity in endometrioma diagnosis, which can be used as an indicator of the diagnostic accuracy of TVS in the tubo-ovarian condition compartment [29]. However, the presence of endometrioma and the tubo-ovarian condition compartment are different. Endometriomas may not cause adhesions by themselves. Also, adhesion of tuba and ovaries may occur without endometrioma. In our results, MRI has $86 \%$ specificity and $92 \%$ sensitivity, which is the only result assessing the tubo-ovarian compartment of the \#ENZIAN classification to date and should be confirmed with further studies.

On the other hand, MRI had low accuracy and low sensitivity in peritoneal lesion detection, which was expected considering the dimension of the peritoneal lesions. Preoperative diagnosis of compartment P does not usually alter the surgical strategy, and therefore small undefined peritoneal lesions could be neglected.

In our study, extragenital organ involvements classified as FU, FB, and FI were not present and therefore not included except for adenomyosis, which is classified as FA. MRI accuracy, sensitivity, and specificity for adenomyosis in this study were $87 \%$, $88 \%$, and $87 \%$, respectively, which is similar to previous studies $[10,30]$.

Limitations of this study are the low number of DIE patients, the retrospective nature of the study, and the lack of rASRM scores. The strengths of this study could be the evaluation of full-time surgery videos by experienced surgeons and the interpretation of MRI by radiologists familiar with DIE cases.

In conclusion, this study demonstrated that utilizing the \#ENZIAN classification in MRI reports has significant sensitivity and specificity in compartments A, B, O, and C. For compartments T and FA, although sensitivity and specificity are not as high as in compartments A, B, C, and $\mathrm{O}$, there was significant sensitivity and specificity for MRI. However, the accuracy of MRI is insufficient in compartment P. Currently our study is the only evaluation of MRI accuracy using the \#ENZIAN classification system, and if future studies support our results, we believe that \#ENZIAN could be used to diagnose DIE lesions preoperatively in MRI.

\section{Declarations}

Author contributions :AFT contributed to concept, study design and manuscript writing. DEl contributed to data collecting and manuscript writing, AAG evaluate the MRI and collected the data, and CK contributed to concept, study design.data analysing and manuscript editing All authors read and approved the submitted manuscript. All authors read and approved the submitted manuscript.

Conflict of interest: The authors declare that they have no confict of interest.

\section{References}


1. Foti PV, Farina R, Palmucci S, Vizzini IAA, Libertini N, Coronella M, Spadola S, Caltabiano R, Iraci M, Basile A, Milone P, Cianci A, Ettorre GC (2018) Endometriosis: clinical features, MR imaging findings and pathologic correlation. Insights Imaging Apr;9(2):149-172

2. Chamié LP, Blasbalg R, Pereira RM, Warmbrand G, Serafini PC (2011) Findings of pelvic endometriosis at transvaginal US, MR imaging, and laparoscopy. Radiographics Jul-Aug;31(4):E77-100

3. Ballard K, Lowton K, Wright J (2006) What's the delay? A qualitative study of women's experiences of reaching a diagnosis of endometriosis. Fertil Steril 86:1296-301 [PubMed: 17070183]

4. Hadfield R, Mardon H, Barlow D, Kennedy S (1996) Delay in the diagnosis of endometriosis: a survey of women from the USA and the UK. Hum Reprod 11:878-80 [PubMed: 8671344]

5. Nnoaham KE, Hummelshoj L, Webster P, d'Hooghe T, de Cicco Nardone F, de Cicco Nardone C, Jenkinson C, Kennedy SH, Zondervan KT (2011) World Endometriosis Research Foundation Global Study of Women's Health consortium. Impact of endometriosis on quality of life and work productivity: a multicenter study across ten countries. Fertil Steril Aug;96(2):366-373.e8

6. Medeiros LR, Rosa MI, Silva BR, Reis ME, Simon CS, Dondossola ER, da Cunha Filho JS (2015) Accuracy of magnetic resonance in deeply infiltrating endometriosis: a systematic review and meta-analysis. Arch Gynecol Obstet Mar;291(3):611-21

7. Johnson NP, Hummelshoj L, Adamson GD, Keckstein J, Taylor HS, Abrao MS, Bush D, Kiesel L, Tamimi R, Sharpe-Timms KL, Rombauts L, Giudice LC (2017) World Endometriosis Society Sao Paulo Consortium. World Endometriosis Society consensus on the classification of endometriosis. Hum Reprod Feb;32(2):315-324

8. Keckstein J, Saridogan E, Ulrich UA, Sillem M, Oppelt P, Schweppe KW, Krentel H, Janschek E, Exacoustos C, Malzoni M, Mueller M, Roman H, Condous G, Forman A, Jansen FW, Bokor A, Simedrea V, Hudelist G (2021) The \#Enzian classification: a comprehensive non-invasive and surgical description system for endometriosis. Acta Obstet Gynecol Scand Jul;100(7):1165-1175

9. Bazot M, Bharwani N, Huchon C, Kinkel K, Cunha TM, Guerra A et al (2017) European society of urogenital radiology (ESUR) guidelines: MR imaging of pelvic endometriosis. Eur Radiol 27(7):2765-2775. https://doi.org/10.1007/s00330-016-4673-z ((Epub 2016/12/05, PubMed PMID: 27921160; PubMed Central PMCID: PMCPMC5486785))

10. Burla L, Scheiner D, Samartzis EP, Seidel S, Eberhard M, Fink D, Boss A, Imesch P (2019) The ENZIAN score as a preoperative MRI-based classification instrument for deep infiltrating endometriosis. Arch Gynecol Obstet Jul;300(1):109-116

11. Burla L, Scheiner D, Hötker AM, Meier A, Fink D, Boss A, Imesch P (2021) Structured manual for MRI assessment of deep infiltrating endometriosis using the ENZIAN classification. Arch Gynecol Obstet Mar;303(3):751-757

12. Di Paola V, Manfredi R, Castelli F, Negrelli R, Mehrabi S, Pozzi Mucelli R (2015) Detection and localization of deep endometriosis by means of MRI and correlation with the ENZIAN score. Eur J Radiol Apr;84(4):568-74

13. Ruffo G, Sartori A, Crippa S et al (2012) Laparoscopic rectal resection for severe endometriosis of the mid and low rectum: technique and operative results. Surg Endosc 26:1035-1040

14. Maytham GD, Dowson HM, Levy B et al (2010) Laparoscopic excision of rectovaginal endometriosis: report of a prospective study and review of the literature. Colorectal Dis 12:1105-1112

15. Koh CE, Juszczyk K, Cooper MJ et al (2012) Management of deeply infiltrating endometriosis involving the rectum. Dis Colon Rectum 55:925-931

16. Tomassetti C, Meuleman C, Vanacker B et al (2009) Lower limb compartment syndrome as a complication of laparoscopic laser surgery for severe endometriosis. Fertil Steril 92:2.038E12-2.038E15

17. Nisenblat V, Bossuyt PM, Farquhar C, Johnson N, Hull ML (2016) Imaging modalities for the non-invasive diagnosis of endometriosis. Cochrane Database Syst Rev Feb 26;2(2):CD009591

18. Andres MP, Borrelli GM, Abrão MS (2018) Endometriosis classification according to pain symptoms: can the ASRM classification be improved? Best Pract Res Clin Obstet Gynaecol Aug;51:111-118

19. (1997) Revised American Society for Reproductive Medicine classification of endometriosis: 1996. Fertil Steril 67(5):817-21

20. Vercellini P, Trespidi L, De Giorgi O, Cortesi I, Parazzini F, Crosignani PG (1996) Endometriosis and pelvic pain: relation to disease stage and localization. Fertil Steril 65(2):299-304

21. Vercellini P, Fedele L, Aimi G, De Giorgi O, Consonni D, Crosignani PG (2006) Reproductive performance, pain recurrence and disease relapse after conservative surgical treatment for endometriosis: the predictive value of the current classification system. Hum Reprod 21(10):2679-85

22. Guzick DS, Silliman NP, Adamson GD, Buttram VC, Canis M, Malinak LR et al (1997) Prediction of pregnancy in infertile women based on the American Society for Reproductive Medicine's revised classification of endometriosis. Fertil Steril 67(5):822-9

23. Vercellini P, Fedele L, Aimi G, Pietropaolo G, Consonni D, Crosignani PG (2007) Association between endometriosis stage, lesion type, patient characteristics and severity of pelvic pain symptoms: a multivariate analysis of over 1000 patients. Hum Reprod 22(1):266-71 
24. Haas D, Oppelt P, Shebl O, Shamiyeh A, Schimetta W, Mayer R (2013) Enzian classification: does it correlate with clinical symptoms and the rASRM score? Acta Obstet Gynecol Scand 92:562-6

25. Mutuku T (2016) Prä-operative Abschätzung einer tief infiltrierenden Darmendometriose mittels Untersuchungsbefund in der ENZIANKlassifikation und der Symptomatik sowie Vergleich mit dem intra- operativen ENZIAN-Befund [The assessment of deep infiltrating endometriosis according to the preoperative investigation and symptoms in comparison to intraoperative findings with the ENZIANClassification]. MD Thesis, Universität UIm

26. Haas D, Chvatal R, Habelsberger A et al (2013) Preoperative planning of surgery for deeply infiltrating endometriosis using the ENZIAN classification. Eur J Obstet Gynecol Reprod Biol 166:99-103

27. Roman H, Moatassim-Drissa S, Marty N et al (2016) Rectal shaving for deep endometriosis infiltrating the rectum: a 5-year continuous retrospective series. Fertil Steril 106:1438-45.e2

28. Alves J, Puga M, Fernandes R, Pinton A, Miranda I, Kovoor E, Wattiez A (2017) Laparoscopic management of ureteral endometriosis and hydronephrosis associated with endometriosis. J Minim Invasive Gynecol Mar-Apr;24(3):466-472

29. Kiesel L, Sourouni M (2019) Diagnosis of endometriosis in the 21st century. Climacteric Jun;22(3):296-302

30. Novellas S, Chassang M, Delotte J, Toullalan O, Chevallier A, Bouaziz J, Chevallier P (2011) MRI characteristics of the uterine junctional zone: from normal to the diagnosis of adenomyosis. AJR Am J Roentgenol 196:1206-1213. doi: 10.2214/AJR.10.4877

\section{Figures}

\section{Figure 1}

Intraoperative aspect of bowel adhesion (U: Uterus, B: Bowel)

\section{Figure 2}

Preoperative MRI of the patient; T2 weighted sagittal plane (BI: bladder, U: uterus, B: bowel, V:vagina, N: hypointense nodüle 